\title{
CONSUMER PARTICIPATION IN POWER MARKET BALANCING
}

\section{A REAL-LIFE STEP TOWARDS SMART GRIDS}

I Per Lund - DOI: 10.1051/epn/2014502

I Chief engineer in System Development at Energinet.dk (the national Danish Transmission System Operator)

With the increasing role of wind and solar power, the power balance authorities are facing a big challenge: How to manage the increasing need for fast balancing power brought on by increased penetration of variable and difficult-to-forecast renewable generation? Could more active participation by the residential customers in managing electricity demand be a smart way to go? 
T his is what the EcoGrid EU field test is all about. The pilot project will introduce a market concept enabling customers to actively take part in the power market and to contribute to the power balancing. The field test is located on the Danish island of Bornholm. It involves 1,900 residential electricity consumers and up to 100 commercial customers, representing almost every tenth electricity consumer on the island.

All test households are equipped with advanced meters, collecting data about their consumption with five-minute resolution. Most of the participants or their electric appliances are "automated", e.g., by various installed home automation systems and software solutions. These solutions enable participants to react to real-time price signals and adjust their electricity consumption based on their predefined comfort preferences. A manual control group of 500 participants can only respond manually to real-time price signals they receive on a dedicated web-based customer feedback system.

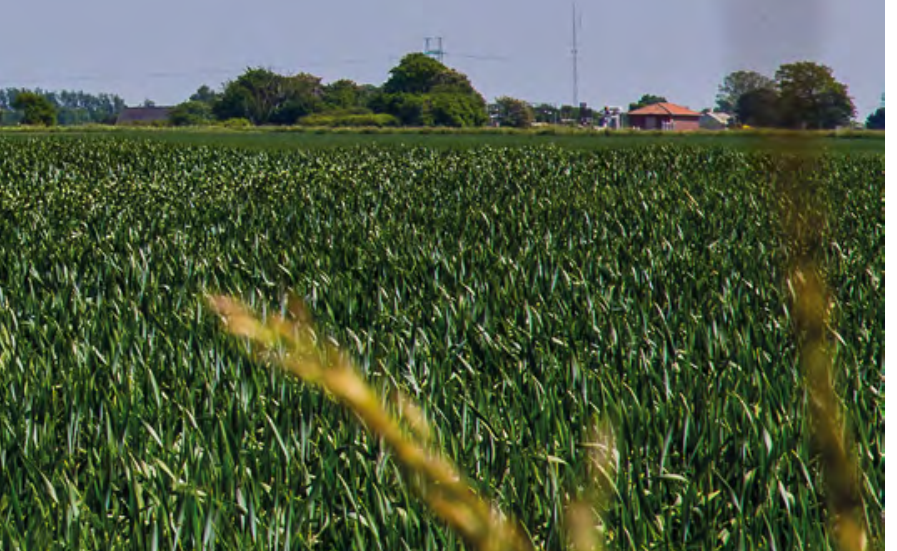

\section{Integration with}

\section{the Nordic electricity system}

What makes Bornholm an interesting test case is that the local distribution system operator (DSO) for the island experiences actual wind power balancing challenges. On a windy day, the wind power on Bornholm can supply up to $30 \mathrm{MW}$, or more than half of the island's peak load of $55 \mathrm{MW}$. But the wind is unreliable, and this variability and unpredictability can have considerable impact on the grid's stability.

At present, cross-border interconnections provide most of the balancing of wind power generation in the Bornholm (and in the Nordic) electricity system. This is possible due to high flexibility in the Nordic hydro-based power system. There will be increased international competition for balancing capacity, and the cost of these resources is therefore expected to increase significantly.

\section{Transition of power systems changes market conditions}

Traditionally, the electricity grid has primarily been designed as a road for moving electricity in a one-way flow from large power generation plants to the customers.

Tomorrow's power system will include a variety of distributed and local energy resources as well as accommodate electric vehicles. This will require two-way flow of both electricity and information, as new technologies enable new forms of generation, supply and use.

Higher amounts of generation capacity from, e.g., wind power requires a more dynamic electricity market, including mobilisation of many more resources that today are either passive or insufficiently integrated into the energy systems. This topic is addressed in the box Modernisation of existing electricity markets.

\section{The EcoGrid ICT platform}

The ICT system and software solutions supporting the EcoGrid real-time market concept do not start from scratch. The ICT architecture is either based on existing software solutions available on the market today, or has been tested in other field situations.

Fig. 3 shows the implementation and the key components of the ICT architecture for real-time price distribution in EcoGrid. Real-time electricity prices are generated at the price-generation module every five minutes.

The ICT implementation consists of a price-generation module and price-distribution components. The price-generation module takes input from i) the Transmission System Operator (TSO), ii) the electricity spot market, iii) historical metering data, and iv) weather forecasts. The generation module sends prices and price forecasts to the price-distribution system, which uses publish-subscribe technology to broadcast the real-time price information to the customers. The EcoGrid ICT concept describes a solution to combine publish-subscribe and so-called Internet Provider multicast technologies (a method for sending 


\section{MODERNISATION OF \\ EXISTING ELECTRICITY MARKETS}

In the existing power market, small-scale generation units and electricity consumers face considerable barriers to supply balancing services. For example, the market operation is currently based on an hourly time resolution that does not reflect the actual dynamics in the power system.

The EcoGrid project introduces a real-time market concept that is based on a 'bidless' market with price announcement beforehand ('ex-ante'). This implies that the final settlement price is determined by prediction of the real-time price responsiveness rather than on explicit bids as known from conventional auction-based power markets. A bidless market minimises the efforts (transaction costs) by small-scale electricity customers or small power generation units, because they must not create bids and schedules, but simply respond to the actual market prices. Small-scale electricity customers typically have the ability to ramp up or ramp down very fast, e.g., by turning on/off their electrical appliances. This gives them the ability to participate in power markets with short time scales and high price volatility.

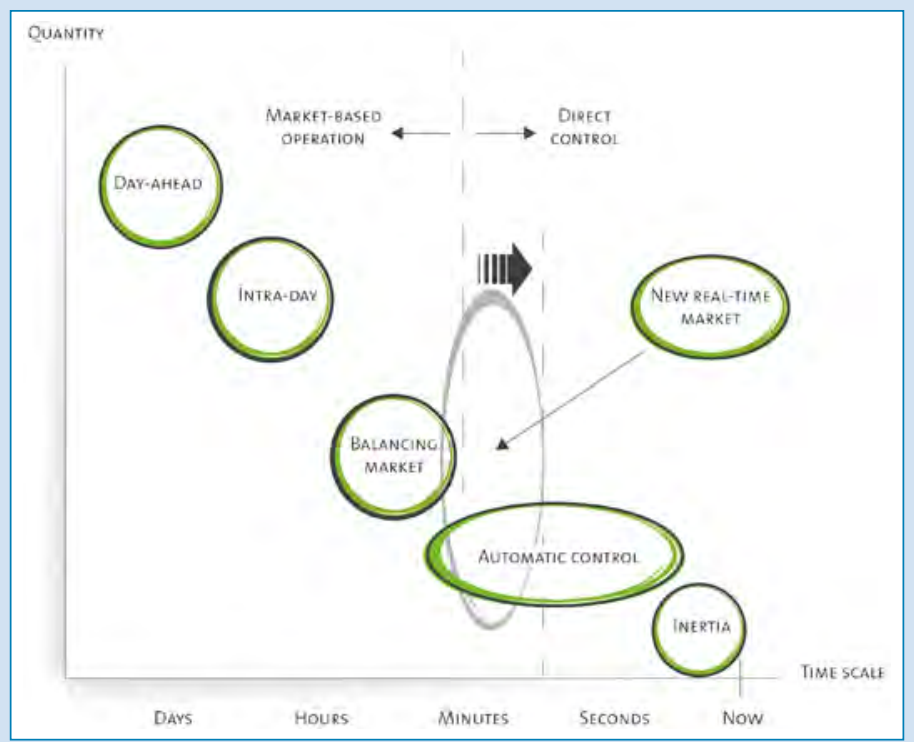

$\triangle F I G$. 1: The new EcoGrid real-time market in the context of existing power-balancing markets and control systems. The five-minute real-time market will extend the market operation closer to real-time and potentially reduce the need for more expensive automatic control resources such as load-frequency control.

From the wholesale market perspective, the EcoGrid concepts imply that the power system is continuously monitored, and that a five-minute price signal is given by, for example, the transmission system operator (TSO) in order to restore the balance of the system. To do so, it is necessary to create reliable forecasts of the expected response to price changes. These will be utilised when computing the marginal price change required to trigger a response of the right size, leading to a proper rebalancing of the system.

As depicted in FIG. 1, introduction of a real-time market will be an obvious step in the further development of the existing electricity wholesale markets and balancing markets that creates more favourable conditions for the future composition of renewable generation and energy resources. This will not necessarily require a replacement of, but an extension of the current market set-up.
Internet Protocol datagrams to a group of interested receivers in a single transmission). This functionality is required for scaling the ICT system up for nationwide use.

Different Internet Service Providers (ISPs) of an area subscribe to the relevant price signals. Within the ISP domains, relevant price streams are multi-casted. This means that the solution can be scaled up, and in case of a massive deployment of the real-time market concept system across the EU member states, it could accommodate millions of residential electricity customers.

Smart devices or end-nodes adjust their planned consumption according to the price information. All households are connected to a smart electricity meter, which measures the power consumption of the device(s) every five minutes. The measured power consumption data are uploaded to the historical metering data repository every 10 minutes.

\section{Vendor-independent solutions}

EcoGrid uses different smart home solutions and different ICT control technologies. This allows for competition and freedom of choice in the hardware and software components. The challenge is of course to prove the 'smart-grid-ness' of these solutions, ensuring that the meters and the equipment installed offer interoperability with the communication technology and software solutions 'behind-the-meter'.

In view of rolling out a smart grid solution, standardisation of the communication and interfaces of the smart home devices need to be taken into account. Another important aspect is the security throughout the system and the use of existing standards for security as well as measures regarding privacy. Based on the demonstration experiences, the industry partners in EcoGrid will provide a framework for standards in communication and device descriptions, so an interoperable system can be assembled.

\section{Recruitment of residential test households}

Before the official recruitment to the EcoGrid field test started, activities were implemented to raise the general awareness of EcoGrid among the public on Bornholm. The target group for the first recruitment activities was the group of (potential) 'first movers' who were informed through media via press releases, invitation to visit Villa Smart (a show room for smart grid solutions on Bornholm). The key message was: "Participate in EcoGrid and make a difference - put Bornholm on the world map ..."

Recruitment of the last participants (involvement of the 'main-stream' group) was challenging. Intensive information campaigns and direct mails were required and more attention was given to the economic benefits of EcoGrid participation.

The recruitment process is considered a success. By August 2013, the objective of 1,900 test households was almost completed. 


\section{2,000 participating customers in the demonstration}

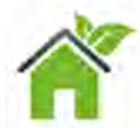

Statistic control group

200 households with smart meters

No acces to specific information or 'smart' equipment

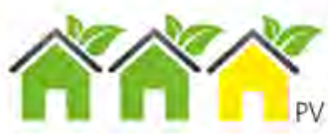

Automatic control group

700 automated households with IBM Green Wave Reality equipment and smart meters

All houses have heat pumps or electric heating all responding autonomously to price signals

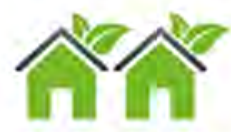

Manual control group

400-500 households with smart meters

Receiving simple market price information

Must move their energy consumption by themselves

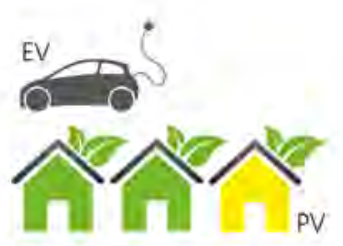

Automatic control group

500 automated households with Siemens equipment and smart meters

All houses have heat pumps, or electric heating all responding to aggregator control

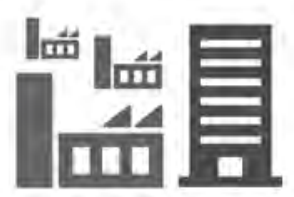

Industry/commercial buildings group

Up to 100 commercial with smart meters

Include also small public customers

Connected smart appliances responsive to control signals

\section{Consumer engagement and involvement}

The establishment of Villa Smart - an EcoGrid showroom/house in Roenne city on Bornholm gives EcoGrid participants the opportunity to experience the technologies that may be installed in their houses to control part of their appliances. At the demonstration house Villa Smart, participants are invited to educational sessions where Oestkraft, the local DSO on Bornholm, offers EcoGrid project-specific advice/education on their participation related to the smart appliances and their customer feedback systems (developed in the EcoGrid project). The website www.ecogridbornholm.dk contains project news stories, FAQ, user guides, EcoGrid videos etc. The international audience can find information at www.eu-ecogrid.net.

The test participants (except for the statistical control group) are introduced to the customer feedback system called 'My EcoGrid'. At'My EcoGrid', the participants can find information about current prices and prognosis for the coming hours. Once every month the participant can find a report informing them of their performance for the past month at 'My EcoGrid', where the EcoGrid cost is compared to the cost of a non-smart grid product.

Last but not least, some of the persons closest to the EcoGrid test participants are the electricians installing the Smart Home equipment in households. It typically takes from one to three hours to install the solutions and make it fit the consumer's wishes - and this is the situation where the customers probably receive the best individual advices.

\section{'Smartness' from the perspective of the individual customer}

The demonstration of EcoGrid takes place in an innovative phase of smart grid - where the attitudes towards smart grid in general are enthusiastic and positive. This period is sometimes referred to as the 'trigger' phase of $\triangle$ FIG. 2: Different participating customer groups.

VFIG. 3: The ECOGrid ICT architecture for price distribution. TSO is the transmission system operator and ISPs are internet service providers.

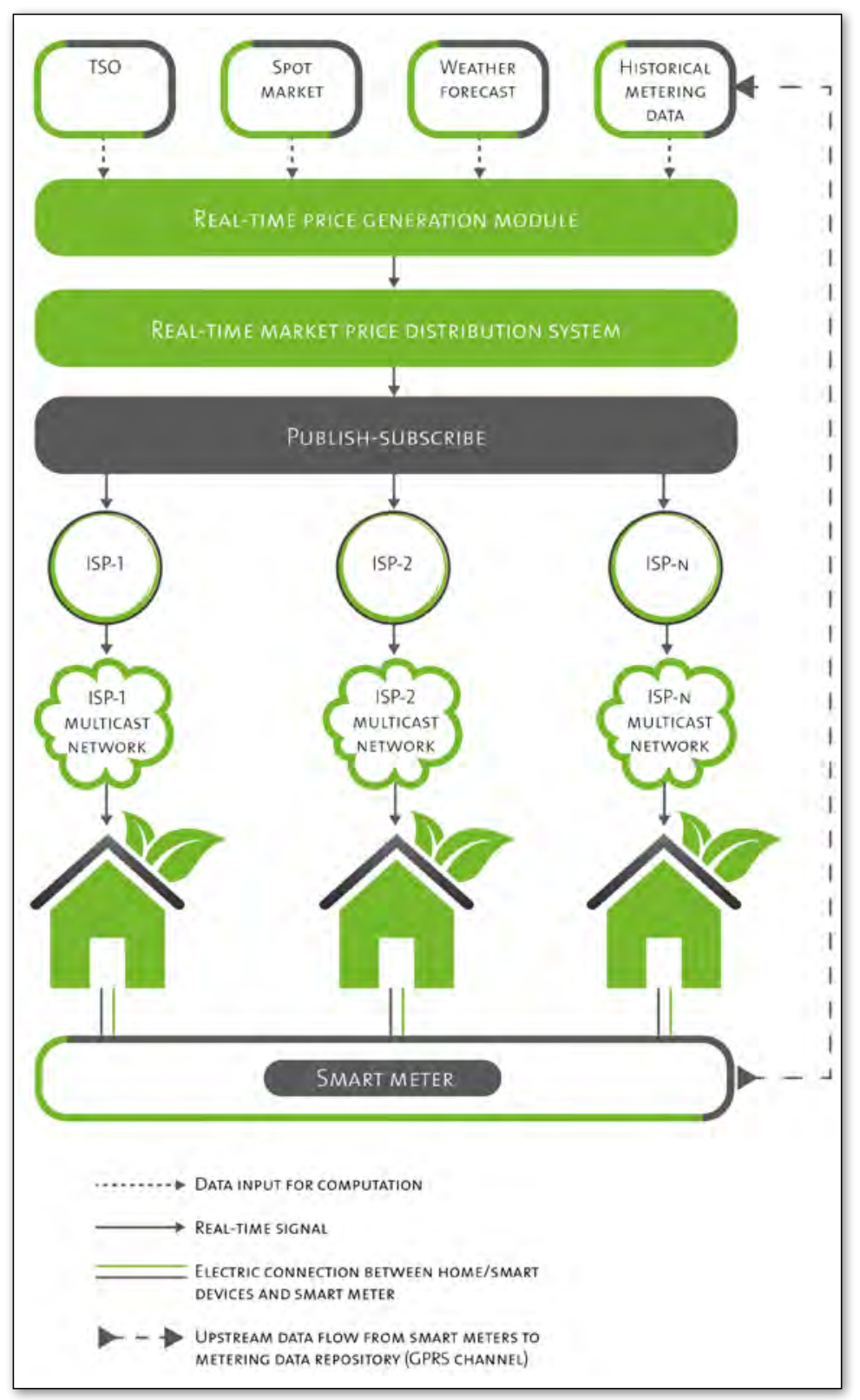


the smart grid. This is a phase that can easily generate attitudes of disillusionment, if the customers do not gain the positive outcome they were expecting.

The smart grid innovations in the EcoGrid project will be 'invisible' for most people. However, what is visible for the test participants is the EcoHome equipment, raising questions like: how does the equipment work and how appealing does it look?

In our experience, you will not only find enthusiasts, first movers or early adopters of new technology/smart grid solutions in a field test involving every tenth residential household on Bornholm. The challenging part in the next test phase (2014-2015) will be to maintain participant involvement.

\section{Will the EcoGrid participants respond to real-time price signals?}

At the time of writing this paper, the EcoGrid project was in a phase with intensive testing and tuning of all the different installed hardware (HW) and software (SW). The different project partners supplying equipment and ICT solutions have to test and tune each of their individual HW/SW parts. Full end-to-end tests of the entire demonstration set-up have to be performed in order to find and remove errors and bugs and to fine-tune for optimal price-elasticity. This is especially the case for the completely novel ICT solutions developed for the project. This is a demanding phase of the project as it involves all server-based and in-house installed SW parts and of course all HW installations in all participating houses. This is also a most critical phase of the project as the resulting level of achievable price-elasticity will be governing the resulting EcoGrid demonstration results.

During the test and tuning phase, a number of intermediate results were recorded. FIG. 4 shows the normalised price-elasticity (red curve) obtained from a group of 140
Siemens-automated houses when subjected to the shown test-price-signal. The black curve shows the simultaneous normalised base-case results from 160 non-automated houses for comparison. The grey area is the $95 \%$ confidence interval of the automated houses if they behaved as the non-automated, i.e., to show true statistical price-elasticity the red curve should be outside the grey area. Hence there were still room for tuning and optimisation.

The test and tuning phase of the EcoGrid project was finalised by mid April 2014. During the summer of 2014, the real-time price generation module of FIG. 3 is to be programmed and implemented in the overall ICT server solution. All necessary closed-loop testing will be conducted and all systems will then be ready for the final EcoGrid demonstration phase to be conducted during the heating season of October 2014 through March 2015.

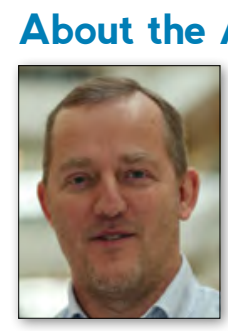

Author

Per Lund is Chief engineer in System Development, Energinet.dk (the national Danish Transmission System Operator). He is a Senior member of IEEE (Institute of Electrical and Electronics Engineers) and a certified European engineer in FEANI EUR ING (Federation of Professional Engineers).

\section{References}

[1] EcoGrid EU: From Design to Implementation - A large scale demonstration of a real-time market place for distributed energy resources, October 2013 (http://eu-ecogrid.net/images/ Documents/131120_ecogrid_popular-report.pdf)

2) EcoGrid EU - A Prototype of European Smart Grids, Presentation by Per Lund at TU/Eindhoven Energy Days, February 2014. https://www.tue.nl/fileadmin/content/ onderzoek/Eindhoven_Energy_Institute_EEl/EnergyDays/Serie_3_2013_2014/Speakers/2014_02_13_Day3/LUND_EcoGrid_EU_TU-Eindhoven_13Feb2014.pdf

\footnotetext{
DFIG. 4: Early indications of price-elasticity of 140 automated Siemens houses. The red curve shows a distinct response to a price increase or decrease. The price at the right scale is an extremely large test price in DKK used during the test and tuning phase of the project.
}

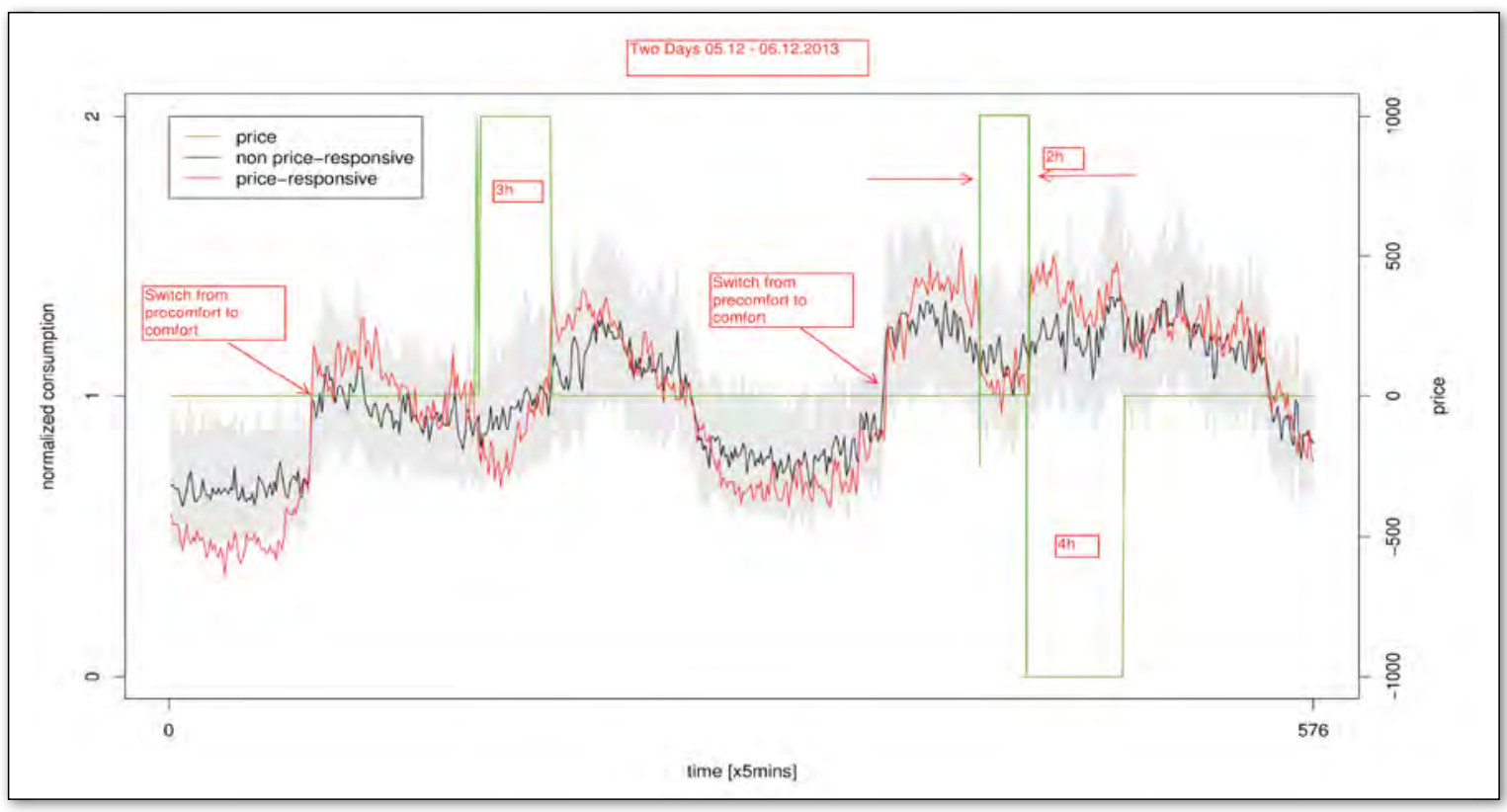

